Trachette L. Jackson

\title{
Vascular tumor growth and treatment: Consequences of polyclonality, competition and dynamic vascular support
}

\author{
Received: 4 August 2000 / Revised version: 13 July 2001 / \\ Published online: 21 February 2002 - (c) Springer-Verlag 2002
}

\begin{abstract}
A mathematical model is presented to describe the evolution of a vascular tumor in response to traditional chemotherapeutic treatment. Particular attention is paid to the effects of a dynamic vascular support system in a tumor comprised of competing cell populations that differ in proliferation rates and drug susceptibility. The model consists of a system of partial differential equations governing intratumoral drug concentration, cancer cell density, and blood vessel density. The balance between cell proliferation and death along with vessel production and destruction within the tumor generates a velocity field which drives the expansion or regression of the neoplasm. Radially symmetric solutions are obtained for the case when only one cell type is present and when the proportion of the tumor occupied by blood vessels remains constant. The stability of these solutions to asymmetric perturbations and to a small semi-drug resistant cell population is then investigated. The analysis shows that drug concentrations which are sufficient to insure eradication of a spherical tumor may be inadequate for the successful treatment of non-spherical tumors. When the drug is continuously infused, linear analysis predicts that whether or not a cure is possible is crucially dependent on the proliferation rate of the semi-resistant cells and on the competitive effect of the sensitive cells on the resistant population. When the blood vessel density is allowed to change dynamically, the model predicts a dramatic increase in the tumor's growth and decrease in its response to therapy.
\end{abstract}

\section{Introduction}

The growth and control of tumors is the subject of considerable clinical, experimental, and theoretical research. The progression of tumors consists of the avascular stage in which nourishment is obtained from the surrounding vasculature; and the vascular stage in which an intratumoral network of blood vessels is established. Avascular tumors are generally harmless in that they are absolutely dependent upon angiogenesis, the formation of blood vessels from the existing vasculature, to grow beyond a nutrient-limited size of a few millimeters in diameter. Vascular tumors, having their own blood supply, have nearly limitless provisions of nutrient and are intrinsically prone to invade the surrounding normal tissue and to

T.L. Jackson: University of Michigan, Department of Mathematics, 525 E. University Ann Arbor, MI 48109-1109, USA. e-mail: tjacks@math. 1sa. umich. edu

Key words or phrases: Mathematical model - Polyclonal tumors - Vascular support 
metastasize to other parts of the body. For these reasons, the growth dynamics and potential control strategies for vascular tumors are of particular interest.

To date most mathematical studies of vascular tumor growth have neglected their spatial heterogeneity and treated the tumor as a spatially-uniform mass which evolves at some prescribed rate such as logistic or Gompertzian. For a review of how spatially-uniform mathematical models capture the in vivo volume growth of murine tumor cell lines, see [37]. Many models of treatment also neglect the spatial environment of the tumor by simulating the cell survival fraction after chemotherapeutic pulses [41], [42]. Recently, however, Jackson and Byrne [28] published a spatially-dependent mathematical model of the response of vascular tumors to chemotherapeutic treatment. In that study, two cell types occupying a neoplastic mass independently of each other (i.e. no direct competition) are considered and it is assumed that the proportion of the tumor occupied by the vascular space remains constant. In this paper, the previous model formulation is extended to investigate the consequences that polyclonality, direct competition, and dynamic vascular support have on a tumor spheroid's response to chemotherapeutic treatment.

This work can be distinguished from that of other authors who have developed spatio-temporal models [1], [2], [3], [6], [7], [24], [25], [36], [46] in that there is no explicit mention of a diffusible nutrient; instead, equations are derived that describe the evolution of the tumor volume, the different types of cancer cells and blood vessels that it contains, and the externally-supplied drug. There are also differences between this study and other investigations of vascular tumor growth [35], [13], [40] as the focus here is on the tumor's response to chemotherapeutic treatment. A modeling approach similar to [6] is adopted to describe the vascular transfer of drug between the blood and tumor. Where they considered the constant transfer of nutrient from the vasculature, we study the effects of increasing vascular density and spatially-dependent transfer of chemotherapeutic drugs into and out of the tumor.

This study concentrates on the spatial properties of tumor composition and structure and the effects they have on successful treatment. In our earlier work [28], we restricted our attention to radially symmetric solutions. Here symmetry assumptions are relaxed in the special case of one cell type and constant vascular support and the way in which radially symmetric solutions respond to asymmetric perturbations is studied. Due to the form of the model equations the natural choice for the perturbations are spherical harmonics. Any modes that are excited indicate how the tumor may invade into its surrounding tissues.

Several authors have studied how these types of perturbations will effect the growth of untreated, avascular multicellular spheroids [25], [12], [11] and [9] has investigated stability of avascular tumors when inhibitors are present. However, to our knowledge, this is the first investigation of how treatment would be affected by such an asymmetric, vascular tumor with realistic drug delivery and elimination. When a dynamic vascular supply is incorporated into the model equations, simulations show a significant increase in the re-growth of the tumor after a single bolus injection compared to the results presented in [28] where the vasculature occupied a constant volume fraction of the tumor. 


\section{Model development}

The objective of this study is to develop a deterministic model that describes tumor reduction due to blood-borne chemotherapy that includes both tumor polyclonality and dynamic vascular support which is under angiogenic control. The tumor is viewed as densely packed, containing blood vessels, $v(\mathbf{r}, t)$, and two types of cells, $n(\mathbf{r}, t), m(\mathbf{r}, t)$ which are in direct competition for the resources necessary for survival and proliferation. Both cell types are susceptible to the drug; however, the drug effects the $m$-cell population to a much lesser degree. Cell movement is produced by the local volume changes that accompany cell proliferation and death which is modified by the presence of the drug, $d(\mathbf{r}, t)$, and blood vessel stimulation and destruction. It is convenient to associate with such movement a local cell velocity $\mathbf{u}(\mathbf{r}, t)$.

The governing equations for drug concentration and the population of tumor cells are derived by applying the principle of conservation of mass to each species and may be written as follows:

$$
\begin{gathered}
\frac{\partial d}{\partial t}+\nabla \cdot(\mathbf{u} d)=D \Delta d+\Gamma(r, v)\left(d_{B}(t)-d\right)-\lambda_{0} d-\lambda_{1} F(d, m, n), \\
\frac{\partial n}{\partial t}+\nabla \cdot(\mathbf{u} n)=D_{n} \Delta n+\alpha_{1}(v) n\left(1-\mu_{1} m\right)-\frac{\beta_{1} d n}{\gamma_{1}+n}, \\
\frac{\partial m}{\partial t}+\nabla \cdot(\mathbf{u} m)=D_{m} \Delta m+\alpha_{2}(v) m\left(1-\mu_{2} n\right)-\frac{\beta_{2} d m}{\gamma_{2}+m}, \\
\frac{\partial v}{\partial t}=-\lambda_{v} v+\delta_{1}(n+m)-\delta_{2} v(n+m),
\end{gathered}
$$

In equation (1), $D$ is the spatially uniform diffusion coefficient of the drug in the tumor. This equation assumes that the discrete vessel source of drug can be replaced by a distributed source term $\left(\Gamma(r, v)\left(d_{B}(t)-d\right)\right)$ as in [30]. This formulation is based on the pore model for transcapillary exchange (for a review see [16], [17]). A further simplification applied to this term is that the ratio of convection to diffusion across the capillary wall is small so that there is no term for the volumetric flow rate out of the vasculature per unit volume of tissue. This simplification is consistent with the observation of increased interstitial pressure which impedes the convection of macromolecules out of the tumor vasculature. The function $d_{B}(t)$ is the prescribed drug concentration in the tumor vasculature and $\Gamma(r, v)$ is the capillary permeability-surface area product per unit volume which is the rate coefficient of blood-tissue transfer. Realistically, the parameter $\Gamma$ may depend on spatial position within the tumor, $\mathbf{r}$. Finally, $\lambda_{0}$ denotes the rate of drug loss due to decay or molecular instability, and $\lambda_{1} F(d, n, m)$ is drug loss due to cellular uptake and metabolism which implies $F(d, m, n)=\frac{\beta_{1} d n}{\gamma_{1}+n}+\frac{\beta_{2} d m}{\gamma_{2}+m}$.

In equations (2) and (3), $D_{n}, D_{m}$ are the assumed constant random motility coefficients of the tumor cells and $\alpha_{1}, \alpha_{2}$ are the net proliferation rates (rate of 
natural cell death subtracted from the rate of cell division) of the susceptible and semi-resistant cells, respectively, in the absence of therapy. It is reasonable to assume that the net proliferation rates are increasing and saturable functions of the blood vessel density. Finally, we assume that the drug's effect on the tumor cells satisfy Michaelis-Menten kinetics.

Equation (4) is based on the model of [19] and describes the effective vascular support provided to the tumor as reflected by the density of cells in the tumor potentially sustainable by it. Tumors gain vascular density via angiogenesis, the formation of blood vessels from the existing supply. The processes involved in tumor-induced angiogenesis are a complex mixture of chemical and mechanical interactions between tumor cells, endothelial cells which line capillary walls, and the extracellular matrix on which they move. The process is believed to be initiated by hypoxic tumor cells producing various tumor angiogenic factors (TAFs) such as vascular endothelial growth factor (VEGF) [26]. TAFs stimulate the proliferation and migration of nearby endothelial cells which eventually form new blood vessels. The second term of (4) represents such stimulation of new vasculature by the tumor through the production of TAFs; whereas, the first term implies that there is a spontaneous loss of functional vasculature at rate $\lambda_{v}$. Experiments have also shown that tumor cells, as well as surrounding normal cells, can produce angiogenic inhibitors. For exapmle thrombospodin-1 (TSP-1) inhibits endothelial cell chemotaxis, aggregation, and proliferation [26]. Finally, the third term in (4) reflects the endogenous inhibition of previously generated vasculature through endothelial cell death or disaggregation [19].

In order to obtain an equation for the velocity, $\mathbf{u}$, we assume that there are no voids within the tumor. Using this assumption, it follows that

$$
V_{c} n+V_{c} m+V_{v} v=1 \quad \text { or } \quad n+m+\frac{V_{v}}{V_{c}} v=\frac{1}{V_{c}}=K \equiv \text { constant }
$$

where $V_{c}$ and $V_{v}$ are the average volumes of a single cell and a single blood vessel, respectively, making the products $V_{c} n, V_{c} m$, and $V_{v} v$ the volume fractions of each component. Adding equations (2), (3), and (4) (multiplied by $\frac{V_{v}}{V_{c}}$ ) leads to the following equation for the local velocity

$$
\begin{aligned}
(n+m) \nabla \cdot \mathbf{u}= & D_{n} \Delta n+D_{m} \Delta m+\alpha_{1} n+\alpha_{2} m-\left(\mu_{1} \alpha_{1}+\mu_{2} \alpha_{2}\right) n m \\
& -\frac{\beta_{1} d n}{\gamma_{1}+n}-\frac{\beta_{2} d m}{\gamma_{2}+m}-\frac{V_{v}}{V_{c}}\left(\lambda_{v} v+\delta_{1} n-\delta_{2} n v\right) .
\end{aligned}
$$

Note that (5) can be used to eliminate one variable from the system and thus equation (6) can be simplified.

In order to assess the tumor's response to the chemotherapeutic treatment it will be important to follow the evolution of the tumor boundary. We do this by denoting the outer surface of the tumor colony by the free boundary $B(\mathbf{r}, t)=0$ where in spherical coordinates $\mathbf{r}=(r, \theta, \phi)$ and

$$
B(\mathbf{r}, t)=r-R(\theta, \phi, t)=0 .
$$


The equation for the motion of a point on $B(\mathbf{r}, t)=0$ is given by

$$
\mathbf{n}_{v} \cdot \frac{d \mathbf{r}}{d t}=\mathbf{u} \cdot \mathbf{n}_{v}
$$

where $\mathbf{n}_{v}$ is an outward unit normal. Equations (1), (6), and (8), subject to the initial and boundary conditions given below, describe the evolution of the tumor velocity, the outer tumor boundary, and the drug concentration within the tumor.

In some of the analysis which follows it will be convenient to reformulate the above problem in terms of a related dependent variable, the internal pressure, $p$. It is assumed that expansion and regression of the tumor mass produce an intratumoral pressure, $p$, and that the bulk velocity of the tumor, $\mathbf{u}$, follows the pressure gradient via Darcy's Law [30], [12]:

$$
\mathbf{u}=-D_{n} \nabla p,
$$

Eliminating $\mathbf{u}$ in favor of $p$, equations (6) and (8) transform to give

$$
\begin{gathered}
\left(K-\frac{V_{v}}{V_{c}} v\right) D_{n} \Delta p \\
=-\left[\alpha_{1} n+\alpha_{2} m-\left(\mu_{1} \alpha_{1}+\mu_{2} \alpha_{2}\right) n m-\frac{\beta_{1} d n}{\gamma_{1}+n}-\frac{\beta_{2} d m}{\gamma_{2}+m}+\frac{V_{v}}{V_{c}} \frac{d v}{d t}\right] \\
\mathbf{n}_{v} \cdot \frac{d \mathbf{r}}{d t}=-D_{n} \nabla p \cdot \mathbf{n}_{v}
\end{gathered}
$$

To complete this system the following initial and boundary conditions are imposed:

$$
\begin{gathered}
n(\mathbf{r}, 0)=n_{0}, \quad m(\mathbf{r}, 0)=m_{0}, \quad v(\mathbf{r}, 0)=v_{0}, \quad d(\mathbf{r}, 0)=0, \quad d_{B}(0)=d_{0}, \\
\frac{\partial n}{\partial r}=\frac{\partial m}{\partial r}=\frac{\partial d}{\partial r}=\frac{\partial p}{\partial r}=0, \quad \text { at } \mathbf{r}=\mathbf{0}, \\
\frac{\partial n}{\partial r}=\frac{\partial m}{\partial r}=0, \quad d=d_{N}(t) \quad p=\alpha_{k} \kappa \quad \text { on } B(\mathbf{r}, t)=0 \\
B(\mathbf{r}, 0)=r-R(\theta, \phi, 0)=0 \quad \text { prescribed. }
\end{gathered}
$$

These conditions imply that initially the tumor has a given composition (cells and vessels) and boundary structure. It is assumed that there is no drug in the tumor tissue at time zero. The concentration of drug on the tumor boundary is assumed to be equal to $d_{N}(t)$, the drug concentration in the surrounding normal tissue which is prescribed. Following [25], at the tumor boundary, the internal pressure field, 
$p$, is proportional to the local curvature, $\kappa$, there. The mean curvature of a surface $r=F(\theta, \phi)$ is given by

$$
\begin{aligned}
\kappa= & -\left(\frac{F^{3}}{2 g^{3 / 2}}\right)\left\{\sin ^{3} \theta\left[\left(F_{\theta}^{2}+F^{2}\right) \frac{F_{\theta}}{F} \frac{\cos \theta}{\sin \theta}+\left(F F_{\theta \theta}-3 F_{\theta}^{2}-2 F^{2}\right)\right]\right. \\
& \left.+\frac{\sin \theta}{F}\left[\left(F_{\theta}^{2}+F^{2}\right) F_{\phi \phi}-3 F F_{\phi}^{2}+F_{\phi}^{2} F_{\theta \theta}-2 F_{\theta} F_{\phi} F_{\theta \phi}+2 F_{\theta} F_{\phi}^{2} \frac{\cos \theta}{\sin \theta}\right]\right\}
\end{aligned}
$$

where subscripts denote partial derivatives and

$$
g(\theta, \phi)=F^{2}\left[F_{\phi}^{2}+\left(F_{\theta}^{2}+F^{2}\right) \sin ^{2} \theta\right] .
$$

\subsection{Nondimensionalization}

The mathematical model is re-scaled in the following manner, denoting nondimensional variables with bars.

$$
\begin{gathered}
d=d_{0} \bar{d}, \quad v=\frac{\bar{v}}{V_{v}}, \quad u=R_{0} T \bar{u}, \quad p=p_{\infty} \bar{p}, \quad n=K \bar{n}, \quad m=K \bar{m}, \\
t=\frac{1}{T} \bar{t}, \quad r=R_{0} \bar{r}, \\
\bar{\lambda}_{0}=\frac{\lambda_{0} R_{0}^{2}}{D}, \quad \bar{\lambda}_{1}=\frac{\lambda_{1} R_{0}^{2}}{D}, \quad \bar{F}(\bar{d}, \bar{n}, \bar{m})=F\left(d_{0} \bar{d}, K \bar{n}, K \bar{m}\right), \quad \bar{\Gamma}=\frac{\Gamma R_{0}^{2}}{D}, \\
\bar{\beta}_{i}=\frac{\beta_{i} d_{0}}{T}, \quad \bar{\gamma}_{i}=\frac{\gamma_{i}}{K_{0}}, \quad \bar{D}_{n} \\
\epsilon=\frac{T R_{0}^{2}}{D}, \quad \sigma_{n}=\frac{D_{n}}{T R_{0}^{2}}, \quad \sigma_{m}=\frac{D_{m}}{T R_{0}^{2}}, \quad v=\frac{D_{n} p_{\infty}}{T R_{0}^{2}} \\
\bar{\lambda}_{k}=\frac{\lambda_{k}}{T}, \quad \bar{\delta}_{1}=\frac{\delta_{1}}{T}, \quad \bar{\delta}_{2}=\frac{\delta_{2} K}{T} .
\end{gathered}
$$

where $T$ is the inverse of the doubling time of the untreated tumor [45] assumed to have only one cell type. After dropping bars for notational convenience, the resulting system of equations to be solved is

$$
\begin{gathered}
\epsilon\left\{\frac{\partial d}{\partial t}+\nabla \cdot(\mathbf{u} d)\right\}=\Delta d+\Gamma\left(d_{B}(t)-d\right)-\lambda_{0} d-\lambda_{1} F(d, n, m), \\
n+m+v=1 \\
\frac{\partial n}{\partial t}+\nabla \cdot(\mathbf{u} n)=\sigma_{n} \Delta n+n\left(1-\mu_{1} m\right)-\frac{\beta_{1} d n}{\gamma_{1}+n}
\end{gathered}
$$




$$
\begin{gathered}
\frac{\partial v}{\partial t}=-\lambda_{v} v+\delta_{1} n-\delta_{2} v n \\
(1-v) \nabla \cdot \mathbf{u}=\sigma_{n} \Delta n+\sigma_{m} \Delta m+n\left(1-\mu_{1} m\right)-\frac{\beta_{1} d n}{\gamma_{1}+1}+\alpha_{2}\left(m-\mu_{2} n\right) \\
-\frac{\beta_{2} d m}{\gamma_{2}+m}-\lambda_{v} v+\delta_{1} n-\delta_{2} v n \\
\mu \Delta p=-\nabla \cdot \mathbf{u}, \\
\mathbf{n} \cdot \frac{d \mathbf{r}}{d t}=-v \nabla p \cdot \mathbf{n} .
\end{gathered}
$$

The initial and boundary conditions scale accordingly.

Inherent in this problem are two timescales of interest: the tumor growth timescale $\left(\approx 1\right.$ week) and the much shorter drug diffusion timescale $\left(R_{0}^{2} / D \approx 1 \mathrm{~min}\right)$ [2]. Accordingly, the nondimensionalization process introduces three small parameters $0<\epsilon=T R_{0}^{2} / D, \sigma_{n}=\frac{D_{n}}{T R_{0}^{2}}, \sigma_{m}=\frac{D_{m}}{T R_{0}^{2}} \ll 1$ into the model equations. Also, since the diffusion coefficient of the cells, $D_{n}$ and $D_{m}$, is much smaller than the diffusion coefficient of the much smaller and more motile drug molecules, $D$, the parameters $\sigma_{n}, \sigma_{m}$ will be at most $O(\epsilon)$. We exploit the appearance of these small parameters in order to construct approximate solutions to which are valid in the limit as $\epsilon, \sigma \rightarrow 0$.

\subsection{Parameter values}

Published data for the treatment of nude mice with the anti-cancer drug doxorubicin is used. Table 1 lists each parameter, its baseline value, and source.

\section{Constant vessel density}

In order to gain some insight into the model behavior, a simplified version of the governing equations is considered. The reduced model is obtained by assuming that the portion of the tumor occupied by cells and the proportion of the tumor occupied by blood vessels remains constant. In section 3.1, we first consider a tumor made up of only one type of cell and obtain radially symmetric solutions. We then investigate the stability of these solutions to asymmetric perturbations in section 3.2. This provides the first such analysis for vascular tumors. Finally, in section 3.3, the semi-drug resistant cell type is re-introduced and the treatment of polyclonal tumors is discussed. The underlying assumption carried through section 3 is that of constant vessel density; in section 4 vessel density is allowed to evolve dynamically. 
Table 1. List of baseline parameter values used in simulations and their sources.

\begin{tabular}{lll}
\hline Parameter & Value & Reference \\
\hline$d_{0}$ & $6 \mathrm{mg} / \mathrm{kg}$ & {$[32]$} \\
$R_{0}$ & $0.4 \mathrm{~cm}$ & {$[32]^{\mathrm{a}}$} \\
$\alpha$ & $\frac{1}{7} \mathrm{day}^{-1}$ & {$[45]^{\mathrm{b}}$} \\
$\lambda$ & $1.9 \mathrm{day}^{-1}$ & {$[4]^{\mathrm{c}}$} \\
$\xi_{1}$ & $60 \mathrm{day}^{-1}$ & {$[43]$} \\
$\xi_{2}$ & $6 \mathrm{day}^{-1}$ & {$[43]^{\mathrm{d}}$} \\
$D$ & $1.7 \mathrm{~cm}^{2} \mathrm{day}^{-1}$ & {$[38]$} \\
$\beta$ & $560 \mathrm{M}^{-1} \mathrm{day}^{-1}$ & {$[18]^{\mathrm{e}}$} \\
$\Gamma$ & $16 \mathrm{day}^{-1}$ & {$[31]^{\mathrm{f}}$} \\
\hline
\end{tabular}

a [32] began chemotherapeutic treatment when tumor volume was approximately $150 \mathrm{~mm}^{3}$ $\left(R_{0} \approx 3.4 \mathrm{~mm}\right.$ ) we chose the slightly larger initial tumor size of $R_{0} \approx 4 \mathrm{~mm}$.

${ }^{\mathrm{b}}$ Estimated from the growth of untreated tumors presented in [45].

${ }^{c}$ Value used in Baxter et al., 1992 to study antibody metabolism.

d There are no reports on the value of $\xi_{2}$ for doxorubicin, we take $\xi_{2}$ to equal to a tenth of the $\xi_{1}$ reported in [43]

${ }^{\mathrm{e}}$ Based on the internalization rate for doxorubicin reported in [18] and the assumption that approximately $10^{6}$ must internalize to initiate cell death.

${ }^{\mathrm{f}}$ Estimated from the vascular permeability of doxorubicin used [31].

\subsection{Treatment of monoclonal symmetric tumors}

Under the assumptions of radially symmetry, one cell type, and constant vessel density, equations (17), (22), and (23), become, to first order (with $\epsilon=0$ ),

$$
\begin{gathered}
0=\frac{D}{r^{2}} \frac{\partial}{\partial r}\left(r^{2} \frac{\partial d}{\partial r}\right)+\Gamma\left(d_{B}(t)-d\right)-\lambda d, \\
\mu \frac{1}{r^{2}} \frac{\partial}{\partial r}\left(r^{2} \frac{d p}{d r}\right)=-(1-\beta d), \\
\frac{d R}{d t}=-v \frac{d p}{d r},
\end{gathered}
$$

where $\beta=\frac{\beta_{1} K^{*}}{\gamma_{1}+K^{*}}, K^{*}=K-v, v$ is constant, and $\lambda=\lambda_{0}+\lambda_{1} \beta$ incorporates drug decay and uptake. Equations (24), (25), and (26) together with (12)-(15) suffice to solve the problem.

To leading order, we solve equation (24) with the appropriate boundary conditions and find

$$
d(r, t)=\left(d_{N}(t)-\frac{\Gamma}{\xi^{2}} d_{B}(t)\right) \frac{R(t) \sinh \xi r}{r \sinh \xi R(t)}+\frac{\Gamma}{\xi^{2}} d_{B}(t)
$$


where $\xi^{2}=\Gamma+\lambda$. Substituting with (27) in (25) and integrating subject to the afore mentioned boundary conditions leads to the following expression for the pressure in the tumor:

$$
\begin{aligned}
p(r, t)= & \frac{\alpha_{k}}{R(t)}-\frac{\left(r^{2}-R^{2}(t)\right)}{6 \mu}\left(1-\frac{\beta \Gamma}{\xi^{2}} d_{B}\right) \\
& +\frac{\beta}{\mu \xi^{2}}\left(d_{N}(t)-\frac{\Gamma}{\xi^{2}} d_{B}(t)\right)\left(\frac{R(t) \sinh \xi r}{r \sinh \xi R(t)}-1\right) .
\end{aligned}
$$

According to (28) the pressure increases toward the center of the tumor where it attains its maximum value which is in agreement with experimental results of [30].

Finally, using (28) an ordinary differential equation which describes the temporal evolution of the tumor radius can be derived. Specifically, substituting (28) into (26) gives

$$
\frac{d R}{d t}=\left(1-\frac{\beta \Gamma}{\xi^{2}} d_{B}(t)\right) \frac{R}{3}-\frac{\beta}{\xi^{2}}\left(d_{N}(t)-\frac{\Gamma}{\xi^{2}} d_{B}(t)\right) \frac{\xi R \cosh \xi R-\sinh \xi R}{R \sinh \xi R} .
$$

If the drug is continuously infused into the bloodstream, the concentrations in the blood and normal tissue will approach steady states, say

$$
\begin{aligned}
& d_{B}(t)=d_{1} \\
& d_{N}(t)=d_{2}
\end{aligned}
$$

This leads to the following steady-state relation for the tumor radius,

$$
\tanh \xi R_{s}=\frac{3 \xi \beta R_{s}\left(d_{2}-\frac{\Gamma}{\xi^{2}} d_{1}\right)}{\left(\xi^{2}-\beta \Gamma d_{1}\right) R_{s}^{2}+3 \beta\left(d_{2}-\frac{\Gamma}{\xi^{2}} d_{1}\right)}
$$

Figure 1 plots $\frac{d R}{d t}$ as a function of $R$ and shows that depending on the parameter values, equation (31) has either one or two real, non-negative roots, $R_{s}=0, R^{*}$. It is clear that $R_{s}=0$ always exits and is stable for large $\beta$. Linearizing (29) around $R=0$ determines stability to time-dependent perturbations occurs if and only if $\beta>1 / d_{N}$. This implies that the tumor can only be eradicated, that is reduced until it is so small that the continuum model ceases to apply, if the drug's effect on its cells is greater than this threshold level. If $\beta<1 / d_{N}$, either treatment will be unsuccessful and the tumor will begin to grow without bound due to the minor effect of the drug or the treatment will be semi-successful in that it has controlled the tumor's growth to the stable, nonzero steady state. These results are valid for timedependent perturbations. The stability of the non-trivial steady state with respect to asymmetric perturbations will be the focus of the next section. 


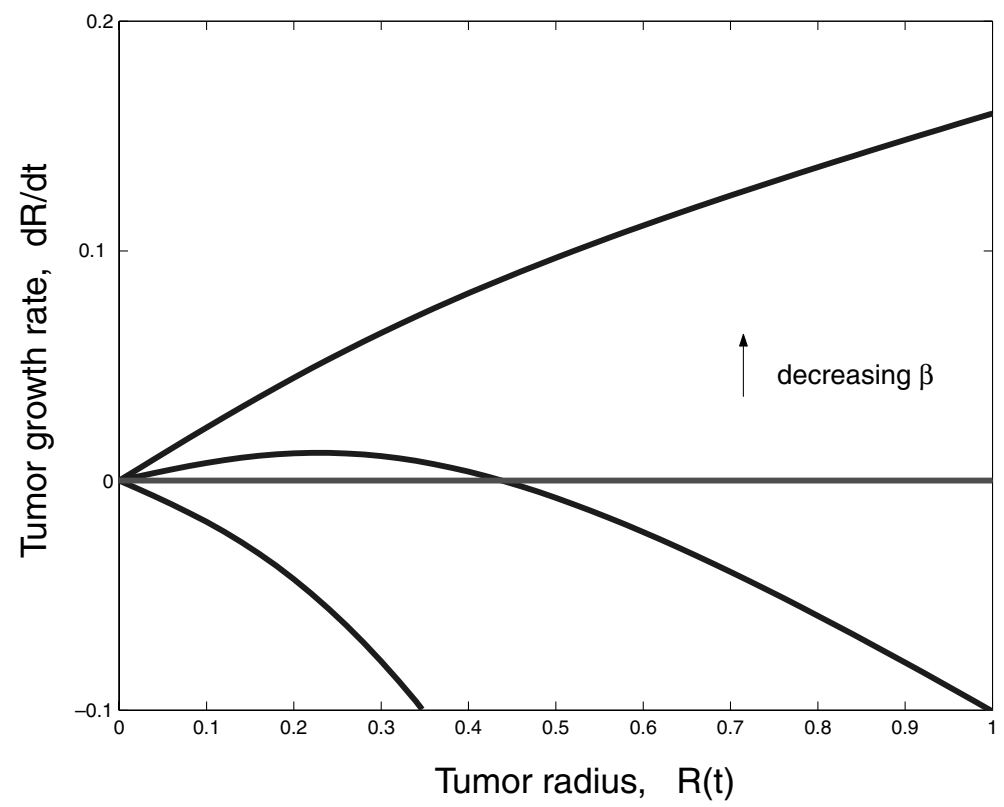

Fig. 1. Plot showing how the tumor's growth rate $\left(\frac{d R}{d t}\right)$ changes with tumor radius $(R)$ for various levels of the drug's effect on tumor cells $(\beta)$. Note that for $\beta$ large, the treatment is successful in controlling the tumor; however, as $\beta$ decreases the drug either reduces the tumor to a nonzero steady state value or is completely ineffective.

\subsection{Treatment of monoclonal asymmetric tumors}

We now derive equations which describe how small perturbations away from radial symmetry evolve in time. This question is investigated in [12] for the case of a growing avascular tumor growth paying particular attention to the role of cell-cell adhesion and [9] took into account the presence of inhibitors. However, to our knowledge, this is the first investigation of how treatment would be affected by such a asymmetries, when vascular tumors are considered.

Although our focus on vascular tumors brings more biological relevance to the model, we adopt the traditional mathematical approach. In order to determine the response of steady, radially symmetric solutions to asymmetric perturbations, we introduce the small parameter $0<\eta \ll 1$ and seek solutions of the form

$$
\begin{aligned}
& d_{\epsilon} \sim d(r, t)+\eta d_{1}(r, \theta, \phi, t) \\
& p_{\epsilon} \sim p(r, t)+\eta p_{1}(r, \theta, \phi, t) \\
& R_{\epsilon} \sim R(t)+\eta R_{1}(r, \theta, \phi, t) .
\end{aligned}
$$

\section{Asymptotic expansions}

Substituting (32) into (24)-(26) and equating to zero coefficients of $O(1)$, the radially symmetric solutions specified by (27)-(29) are recovered. Continuing to $O(\eta)$, 
the following equations governing the evolution of $\left(d_{1}, p_{1}, R_{1}\right)$ are obtained,

$$
\begin{gathered}
\Delta d_{1}-\xi^{2} d=0 \\
v \Delta p_{1}=\beta d_{1} \\
\frac{\partial R_{1}}{\partial t}=-\left.v\left(\frac{\partial p_{1}}{\partial r}+R_{1} \frac{\partial^{2} p}{\partial r^{2}}\right)\right|_{r=R} \\
d_{1}(R, \theta, \phi, t)=-\left.R_{1} \frac{\partial d}{\partial r}\right|_{r=R} \\
p_{1}(R, \theta, \phi, t)=-\left.R_{1} \frac{\partial p}{\partial r}\right|_{r=R}-\frac{\alpha_{k}}{2 R^{2}}\left(\mathcal{L}\left(R_{1}\right)+2 R_{1}\right) \\
R_{1}(\theta, \phi, 0)=\text { prescribed }
\end{gathered}
$$

where in equation $(37), \mathcal{L}(\cdot)$ denotes the angular component of the Laplacian operator

$$
\Delta f=\frac{1}{r^{2}} \frac{\partial}{\partial r}\left(r^{2} \frac{\partial f}{\partial r}\right)+\frac{\mathcal{L}(f)}{r^{2}}
$$

and

$$
\mathcal{L}(f)=\frac{1}{\sin \theta} \frac{\partial}{\partial \theta}\left(\sin \theta \frac{\partial f}{\partial \theta}\right)+\frac{1}{\sin ^{2} \theta} \frac{\partial^{2} f}{\partial \phi^{2}} .
$$

We seek solutions of the form

$$
\begin{aligned}
d_{1} & =\Sigma \xi^{2} \chi_{l m}(t) h(r) r^{l} Y_{l m}(\theta, \phi), \\
p_{1} & =\Sigma\left\{\Pi_{l m}(t)+\frac{\beta}{v} h(r) \chi_{l m}\right\} r^{l} Y_{l m}(\theta, \phi), \\
R_{1} & =\Sigma \rho_{l m}(t) Y_{l m}(\theta, \phi),
\end{aligned}
$$

where the spherical harmonics, $Y_{l m}$, satisfy

$$
\mathcal{L}\left(Y_{l m}\right)=-l(l+1) Y_{l m}
$$

so that $\Delta\left(r^{l} Y_{l m}\right)=0$ and the function $h$ is the solution of

$$
\frac{d^{2} h}{d r^{2}}+\frac{2(l+1)}{r} \frac{d h}{d r}-\xi^{2} h=0 ;
$$

therefore,

$$
h=r^{-(l+1 / 2)} J_{l+1 / 2}(i \xi r)
$$

where $J$ is the Bessel function of the first kind. 
Expressions relating the coefficients $\chi_{l m}$ and $\Pi_{l m}$ to $\rho_{l m}$ are obtained by imposing (36) and (37) and exploiting the orthogonality of the spherical harmonics:

$$
\begin{aligned}
\xi^{2} \chi_{l m} h(R) R^{l}=-\rho_{l m}\left(d_{2}-\frac{\Gamma}{\xi^{2}} d_{1}\right)\left(\frac{\xi R \cosh \xi R-\sinh \xi R}{R \sinh \xi R}\right) \\
\Pi_{l m} R^{l}=-\frac{\beta}{v} h(R) R^{l} \Xi_{l m} \\
+\rho_{l m}\left\{\frac{R}{3 \mu}-\frac{\beta\left(d_{2}-\frac{\Gamma}{\xi^{2}} d_{1}\right)}{\mu \xi^{2}}\left(\frac{\xi R \cosh \xi R-\sinh \xi R}{R \sinh \xi R}\right)\right\} \\
+\frac{\alpha_{k}^{2}}{2 R^{2}}(l-1)(l-2) \rho_{l m}
\end{aligned}
$$

Substituting (45) and (46) into (35) gives

$$
\begin{aligned}
\frac{1}{\rho_{l m}} \frac{d \rho_{l m}}{d t}= & -\frac{a}{3}(l-1)-\frac{\beta}{\xi^{2}}\left(d_{2}-\frac{\Gamma}{\xi^{2}} d_{1}\right)\left[\xi^{2} u-(l+2)\left(\frac{\xi R \cosh \xi R-\sinh \xi R}{R^{2} \sinh \xi R}\right)\right] \\
& -\frac{\mu \alpha_{k}^{2}}{2 R^{3}} l(l-1)(l-2)+\frac{\beta}{\xi^{2}}\left(d_{2}-\frac{\Gamma}{\xi^{2}} d_{1}\right) \\
& \left.\left(\frac{\xi R^{2} \cosh \xi R-\sinh \xi R}{R \sinh \xi R}\right) \frac{1}{h} \frac{d h}{d r}\right|_{r=R}
\end{aligned}
$$

where $a=1-\frac{\beta \Gamma}{\xi^{2}}$. Note that the system is insensitive to perturbations involving the first spherical harmonic as equation (47) reduces when $l=1$ to

$$
\frac{1}{\rho_{l m}} \frac{d \rho_{l m}}{d t}=-\left.\frac{a}{3}(l-1)\right|_{l=1}-\left.\frac{\mu \alpha_{k}^{2}}{2 R^{3}} l(l-1)(l-2)\right|_{l=1}=0 .
$$

This is to be expected since modes having $l=1$ correspond to a translation of the coordinate axes.

As other authors have found [25], [9], [11],[12], the temporal evolution of the radial coefficients $\rho_{l m}$ are independent of $m$. Since $R(t)$ is a dynamic variable, particular modes $Y_{l m}$ may be excited for certain ranges of $R$ and damped for others. For easy comparison with [12], Figure 2 shows how $\frac{1}{\rho_{l m}} \frac{d \rho_{l m}}{d t}$ changes with $R$ for several choices of $l$ when all other parameters are held fixed. Also $\frac{d R}{d t}$ is plotted as a function of $R$ which shows that the nonzero steady state, $R^{*}$, corresponding to control of the tumor is stable to time dependent perturbations. However, the growth rates of the asymmetric modes clearly demonstrate that this steady state is unstable to certain modes of asymmetric perturbations. This result suggests intratumoral drug concentrations which are adequate for the successful treatment of spherical tumors may be inadequate for non-spherical tumors. The unstable modes represent the unsuccessful treatment and invasive growth of an asymmetric tumor under the same conditions which resulted in the control of a spherical tumor.

Figure 3 focuses on the stability of the nontrivial steady state, $R_{S}$, given by (31) with respect to asymmetric perturbations. For each value of $R_{s}$, we plot the range 


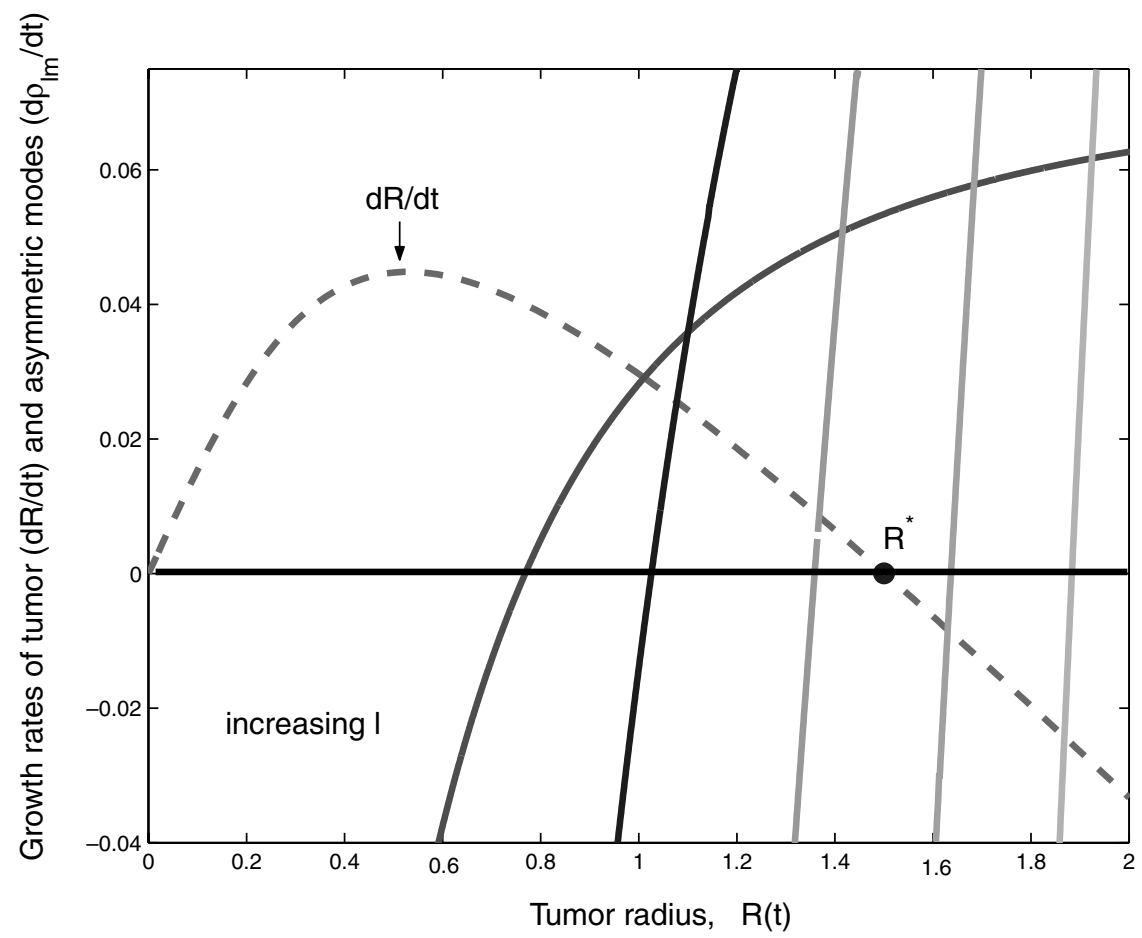

Fig. 2. The growth rate of asymmetric modes $\left(\frac{d \rho_{l m}}{d t}\right)$ is plotted against the tumor radius for various choices of $l(l=1,2,5,10,15,20)$. The growth rate of the tumor is also plotted against $R$ to show when asymmetric instability arises.

of $l$ for which the steady state is unstable. As $R_{S}$ decreases, which corresponds to increasing the drug's effect on the tumor cells $(\beta)$, the range of instability becomes progressively smaller until the steady state is eventually stable to all modes of the asymmetric perturbations.

\subsection{Treatment of polyclonal symmetric tumors}

The preceding analysis gives some insight into conditions which allow for the successful treatment of monoclonal, vascular tumors. In this section, the second cell population is re-introduced into the model $(m>0)$ and it is assumed that these cells are less responsive to the therapy. Being dependent on the cell densities, the intratumoral drug profiles are altered by the re-introduction of $m$ into the model and (24) now has the form

$$
0=\frac{D}{r^{2}} \frac{\partial}{\partial r}\left(r^{2} \frac{\partial d}{\partial r}\right)+\Gamma\left(d_{B}(t)-d\right)-\lambda_{0} d-\lambda_{1}\left[\frac{\beta_{1}}{\gamma_{1}} d n+\frac{\beta_{2}}{\gamma_{2}} d(1-n)\right]
$$




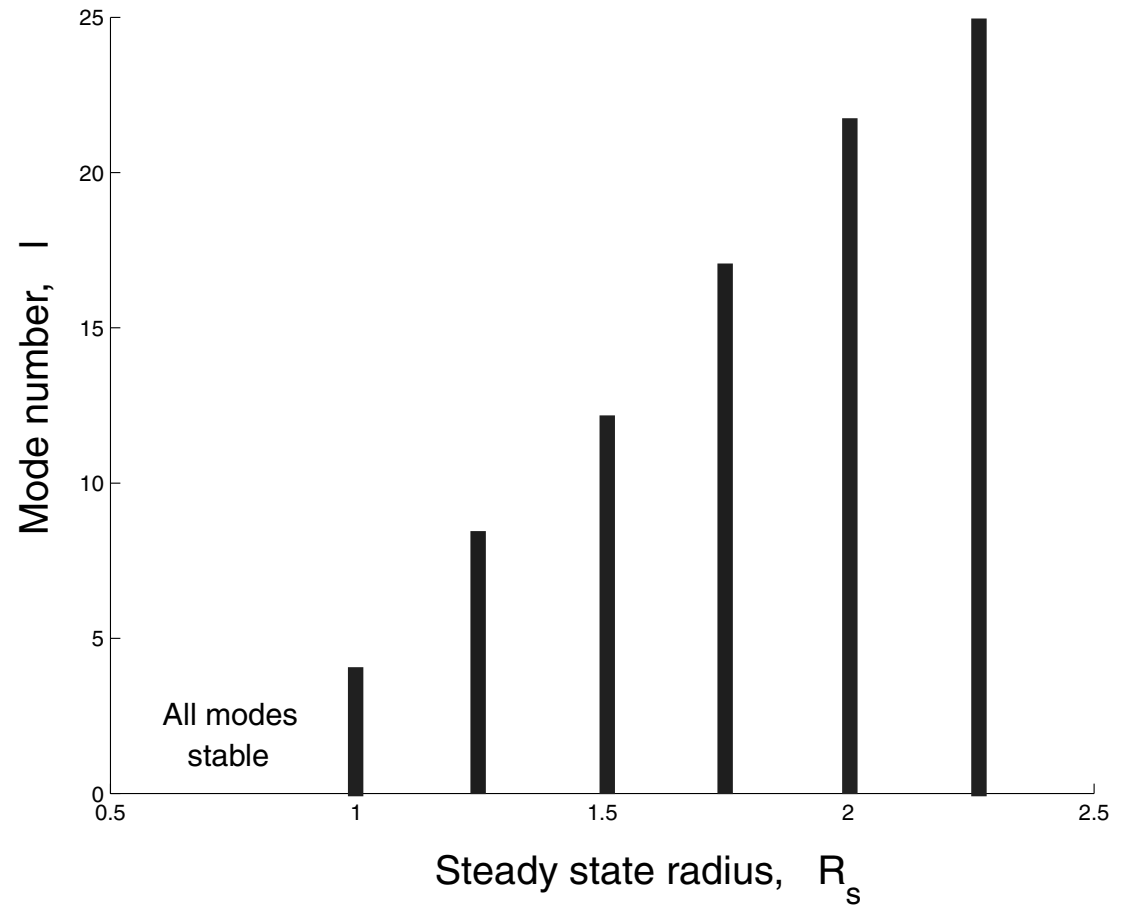

Fig. 3. Diagram depicting those asymmetric modes $\left(\rho_{l m}\right)$ to which the nontrivial steady state radius $\left(R_{s}\right)$ given by (29) is unstable.

To first order, the non-dimensional equations which describe the evolution of the $n$-cells, the cell velocity and the tumor radius are:

$$
\begin{gathered}
\frac{\partial n}{\partial t}+\frac{1}{r^{2}} \frac{\partial}{\partial r}\left(r^{2} u n\right)=n-\mu_{1} n(1-n)-\frac{\beta_{1}}{\gamma_{1}} d n \\
\frac{1}{r^{2}} \frac{\partial}{\partial r}\left(r^{2} u\right)=n-\mu_{1} n(1-n)+\alpha_{2}(1-n)-\mu_{2}(1-n) n-\frac{\beta_{1}}{\gamma_{1}} d n-\frac{\beta_{2}}{\gamma_{2}} d(1-n) \\
\frac{d R}{d t}=u(R, t)
\end{gathered}
$$

where it has been assumed that $\gamma_{i}, \delta_{i}, \beta_{i} \gg 1$ with $\frac{\beta_{i}}{\gamma_{i}} \sim O(1)$ which means the response of both cell types to the treatment is linear. Recall, by incompressibility, the density of the $m$-cells is known in terms of the density of the $n$-cells: $m=1-n$.

Now if the density of drug-resistant cells is small, we introduce the small parameter $0<\eta \ll 1$ and seek solutions of the form

$$
n=1-\eta \tilde{n}, \quad m=\eta \tilde{m}, \quad u=u_{0}+\eta u_{1}, \quad R=R_{0}+\eta R_{1} .
$$


Substituting (53) into (50) and (51) and equating to zero terms of $O\left(\eta^{n}\right)$ allow for the determination $u_{0}, u_{1}, R_{0}, R_{1}$ and $\tilde{n}$. Of particular interest is establishing conditions under which the drug resistant population grows or decays.

Assuming constant drug concentration, $d_{c}$, within the tumor mass results in the following solutions

$$
\left.\begin{array}{l}
u_{0}(r, t)=\frac{r}{3}\left(1-\frac{\beta_{1}}{\gamma_{1}} d_{c}\right) \\
\tilde{n}(r, t)=\tilde{n}_{0} \exp \left\{\left[d_{c}\left(\frac{\beta_{1}}{\gamma_{1}}-\frac{\beta_{2}}{\gamma_{2}}\right)-\left(1-\alpha_{2}+\mu_{2}\right)\right] t\right\} \\
u_{1}=\frac{\tilde{p}_{0}\left[\left(\alpha_{2}-\frac{\beta_{2}}{\gamma_{2}} d_{c}\right)-\left(1-\frac{\beta_{1}}{\gamma_{1}} d_{c}\right)-\mu_{2}\right] r}{3} \exp \left\{\left[d_{c}\left(\frac{\beta_{1}}{\gamma_{1}}-\frac{\beta_{2}}{\gamma_{2}}\right)-\left(1-\alpha_{2}+\mu_{2}\right)\right] t\right\}
\end{array}\right\}
$$

Equation (54) is valid for

$$
0<r<R_{0}(t)=e^{\left(1-\frac{\beta_{1}}{\gamma_{1}} d_{c}\right) t / 3}
$$

From (54) we see that perturbations in the resistant cell density will grow if $d_{c}>$ $\frac{1-\alpha_{2}+\mu_{2}}{\frac{\beta_{1}}{\gamma_{1}}-\frac{\beta_{2}}{\gamma_{2}}}$ and decay otherwise. Also, the tumor will regress, to first order, if $d_{c}>\frac{\gamma_{1}}{\beta_{1}}$, but perturbations will decay if $d_{c}<\frac{1-\alpha_{2}+\mu_{2}}{\frac{\beta_{1}}{\gamma_{1}}-\frac{\beta_{2}}{\gamma_{2}}}$. Now the two limiting curves which mark the transition between growth and decay of the perturbations intersect when $\beta_{2}=\beta^{*}=\frac{\left(\alpha_{2}-\mu_{2}\right) \beta_{1} \gamma_{2}}{\gamma_{1}}$ giving a threshold for the drug's effect on the resistant cell population. Notice that when there is no competitive effect of the susceptible cells on the resistant population, i.e. $\mu_{2}=0$, the threshold drug effect $\beta^{*}$ found in [28] is recovered. Further, $\mu_{2}>0$ reduces the strength of the drug required to successfully treat the polyclonal tumor. Also, in [28] it is shown that as long as the resistant cells proliferate less rapidly than the susceptible cells $\left(\alpha_{2}<1\right)$ there is region in parameter space where all perturbations decay and the tumor regresses. When $\mu_{2}>0$, this condition becomes $\alpha_{2}<1+\mu_{2}$, which implies that the resistant cells can proliferate faster without effecting the final successful outcome.

The polyclonal tumor's response to treatment as determined by (49)-(52) is plotted against time in Figure 4. The tumor is initially composed of $75 \%$ n-cells and $25 \% \mathrm{~m}$-cells. As the competition parameters $\mu_{1}, \mu_{2}$ increase, the tumor's response to therapy improves. Figure 4A shows that the best results are seen when the competitive effect of the n-cells on the m-cells, $\mu_{2}$, is much stronger than the competitive effect of the m-cells on the n-cells, $\mu_{1}$. The fact that this significant reduction in growth is due to treatment is demonstrated in Figure 4B where the untreated and best treatment scenarios are plotted vs. time.

\section{Dynamic blood vessel density}

The analysis and simulations presented in section 3 assume that the tumor is initially vascular and that the proportion of the spheroid consisting of blood vessels remains constant. In this section equation (20) is re-introduced into the model and the effects of a temporally varying vascular support system on tumor growth and 

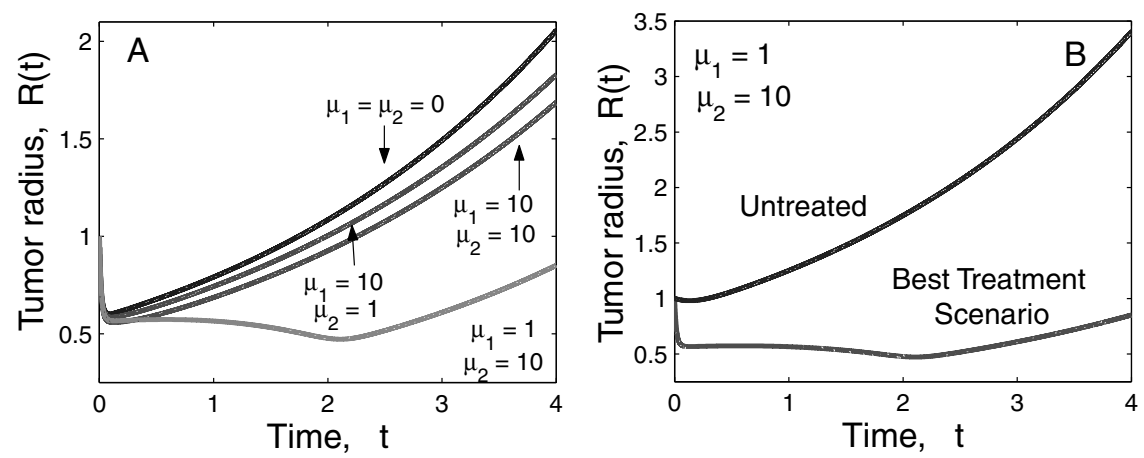

Fig. 4. The tumor's response to therapy for various competitive effects is plotted against time in (A) and an untreated tumor is compared with the best treatment scenario in (B). The initial conditions are $n_{0}=0.75, m_{0}=0.25$.

treatment is investigated. Specifically, in section 4.1, a tumor composed of only one cell type is considered and in section 4.2, the semi-drug resistant tumor cell population is re-introduced.

\subsection{Monoclonal tumors}

The equations for a tumor containing only one type of cell and a dynamic vascular support system are as follows:

$$
\begin{gathered}
0=\frac{D}{r^{2}} \frac{\partial}{\partial r}\left(r^{2} \frac{\partial d}{\partial r}\right)+\Gamma(v)\left(d_{B}(t)-d\right)-\lambda d n \\
\frac{\partial n}{\partial t}+\frac{1}{r^{2}} \frac{\partial}{\partial r}\left(r^{2} u n\right)=n-\frac{\beta_{1}}{\gamma_{1}} d n, \\
\frac{\partial v}{\partial t}=-\lambda_{v} v+\delta_{1} n-\delta_{2} v n, \\
n \frac{1}{r^{2}} \frac{\partial}{\partial r}\left(r^{2} u\right)=n-\frac{\beta_{1}}{\gamma_{1}} d n-\lambda_{v} v+\delta_{1} n-\delta_{2} v n, \\
\frac{d R}{d t}=u(R, t) .
\end{gathered}
$$

where $\lambda=\lambda_{0}+\lambda_{1} \frac{\beta_{1}}{\gamma_{1}}$ and $\Gamma(v)=\Gamma_{0} v$. Recalling that $n+v=1$ allows the for the following analytical solution of equations (57) and (58)

$$
n(r, t)=\frac{1}{\delta_{2}}\left[\sqrt{b^{2}+4 \lambda \delta_{2}} \tanh \left\{\frac{t}{2} \sqrt{b^{2}+4 \lambda \delta_{2}}+c\right\}-b\right]
$$




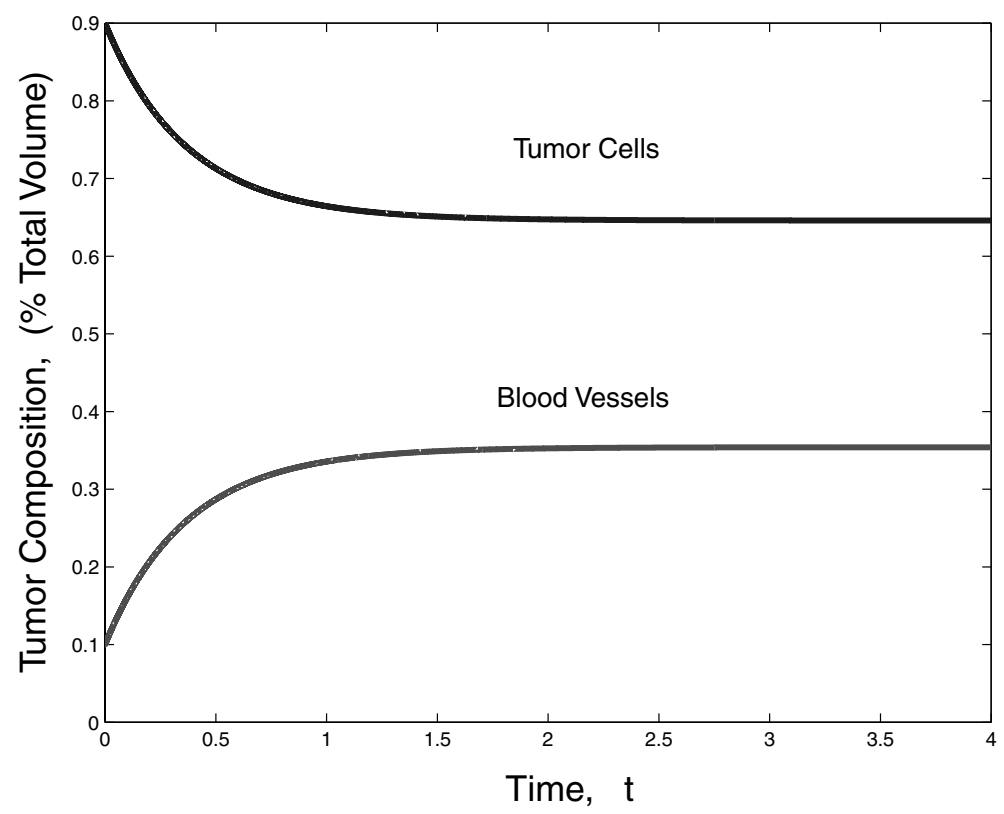

Fig. 5. Plot of temporal changes in tumor composition. The upper curve plots the volume fraction of tumor cells which settle to a steady state value of 0.65 after approximately one week. The lower curve shows the temporal increase in the vascular support until steady state is reached.

where $b=\delta_{1}+\lambda-\delta_{2}$ and $c$ is determined from the initial condition. This solution implies that the densities of tumor cells and blood vessels will approach the steady state values of

$$
\begin{aligned}
& n^{*}=\frac{1}{\delta_{2}}\left[\sqrt{b^{2}+4 \lambda \delta_{2}}-b\right] \approx 0.66 \\
& v^{*}=1-n^{*} \approx 0.34
\end{aligned}
$$

Temporal changes in the composition of the tumor when dynamic vascular support is included are shown in Figure 5. For constant initial conditions $n_{0}=0.9$ and $v_{0}=0.1$ we see that steady state is reached by approximately $t=1$.

This increase in vessel density has a substantial impact on the tumor's growth and susceptibility to chemotherapeutic treatment. From (59) we see that including the vascular support results in a net increase in the tumor velocity when $v<\frac{\delta_{1}}{\frac{\lambda}{n}+\delta_{2}}$. This condition holds as long as the initial volume fraction of tumor cells is greater than the steady state value, i.e. $n_{0}>n^{*}$. Figure 6 compares the tumor's response to chemotherapeutic treatment when dynamic vascular support is incorporated to the case where blood vessels make up a constant proportion of the tumor. When the blood vessel density changes with time, not only is the tumor less responsive to chemotherapeutic treatment, as the drug's effect wears off, the re-growth of the tumor with dynamic vascular support is faster. 


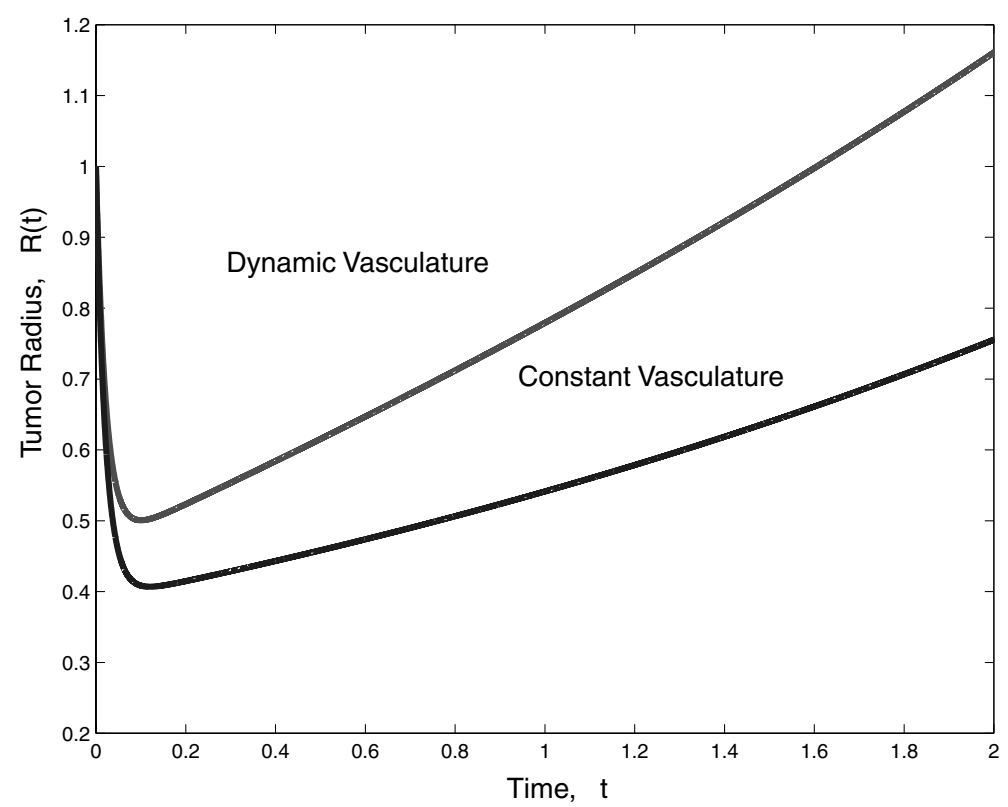

Fig. 6. Tumor radius plotted as a function of time for dynamic vessel density given by (58) with $v_{0}=0.1$ (in this case vessels density approaches $v^{*}=0.34$ as shown in Figure 5) and for constant vessel density, $v=0.1$.

\subsection{Polyclonal tumors}

In this section we consider two competing cell populations within a tumor containing a dynamic vascular support system. In this case we have

$$
\begin{gathered}
0=\frac{D}{r^{2}} \frac{\partial}{\partial r}\left(r^{2} \frac{\partial d}{\partial r}\right)+\Gamma(v)\left(d_{B}(t)-d\right)-\lambda_{1}\left[\frac{\beta_{1}}{\gamma_{1}} d n+\frac{\beta_{2}}{\gamma_{2}} d m\right] \\
\frac{\partial n}{\partial t}+\frac{1}{r^{2}} \frac{\partial}{\partial r}\left(r^{2} u n\right)=n\left(1-\mu_{1} m\right)-\frac{\beta_{1}}{\gamma_{1}} d n \\
\frac{\partial m}{\partial t}+\frac{1}{r^{2}} \frac{\partial}{\partial r}\left(r^{2} u m\right)=m\left(1-\mu_{2} n\right)-\frac{\beta_{2}}{\gamma_{2}} d m \\
\frac{\partial v}{\partial t}=-\lambda_{v} v+\delta_{1}(n+m)-\delta_{2} v(n+m) \\
(n+m) \frac{1}{r^{2}} \frac{\partial}{\partial r}\left(r^{2} u\right)=n\left(1-\mu_{1} m\right)-\frac{\beta_{1}}{\gamma_{1}} d n+m\left(1-\mu_{2} n\right)-\frac{\beta_{2}}{\gamma_{2}} d m+\frac{d v}{d t}
\end{gathered}
$$




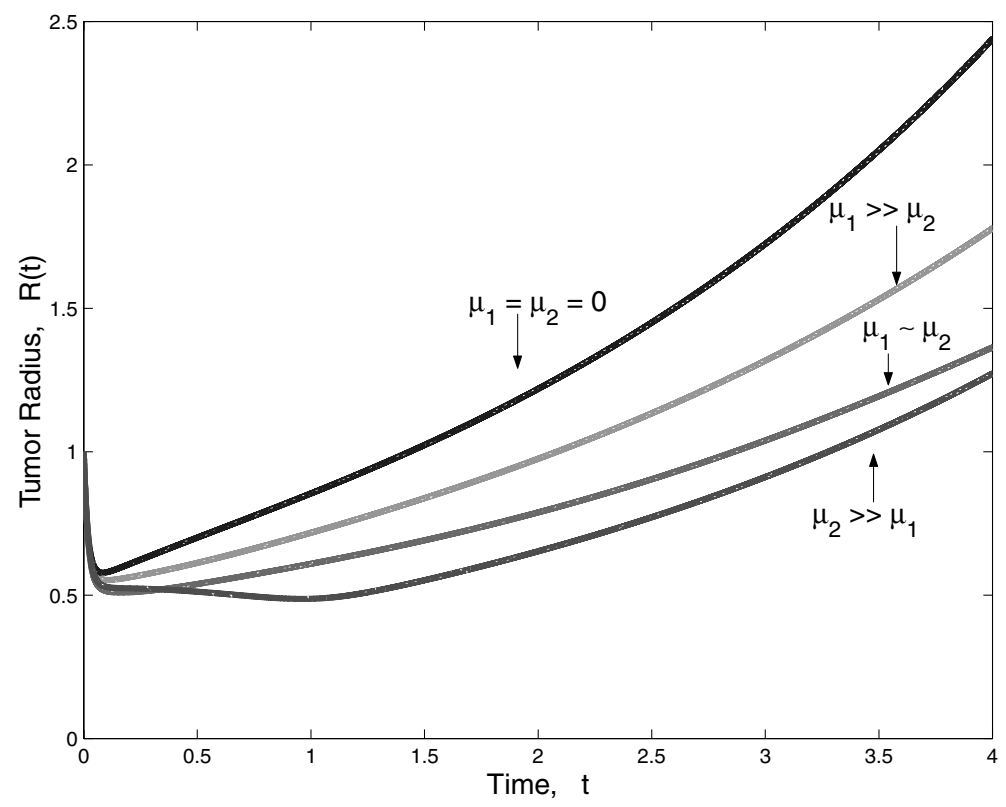

Fig. 7. The tumor's response to therapy for various competitive effects is plotted against time when dynamic vascular support governed by (66) is included. The initial conditions are $n_{0}=0.75, m_{0}=0.15, v_{0}=0.1$.

$$
\frac{d R}{d t}=u(R, t)
$$

From equation (66) it is clear that the density of the blood vessels and hence the density of total cell population, $n+m$, is the same as in the previous section. Therefore, it is the changes in the densities of each individual cell type and the effect on treatment that are determined. Recall, that in section 3.3 (Figure 4), chemotherapeutic treatment was most successful when $\mu_{2} \gg \mu_{1}$. Figure 7 shows that this result is a bit more complicated when dynamic vascular support is included into the model. In this case, although $\mu_{2} \gg \mu_{1}$ results in prolonged control of tumor growth when compared to $\mu_{1} \sim \mu_{2} \gg 1$, eventually the re-growth of the tumor with $\mu_{2} \gg \mu_{1}$ is faster than the tumor with $\mu_{1} \sim \mu_{2} \gg 1$.

Figure 8 depicts the tumor configuration during treatment with $\mu_{2} \gg \mu_{1}$. The percentage of the tumor made up of drug-sensitive cells is plotted versus the scaled tumor radius in figure 8A. Because there is an additional source of drug from the normal tissue surrounding the tumor at $r^{*}=1$, we see a greater reduction of sensitive cells as we move toward the periphery. Figure 8B plots the percentage of semi-drug resistant cells in the tumor and $8 \mathrm{~B}$ shows the fraction of blood vessels. It is important to note that in this best treatment scenario, the drug sensitive cells out compete the semi-resistant cell type.

Figure 9 compares a polyclonal tumor's response to therapy when vascular support makes up a constant proportion of the tumor to the case when vessel density changes with time. Here the dramatic effect that dynamic vascular support can have 


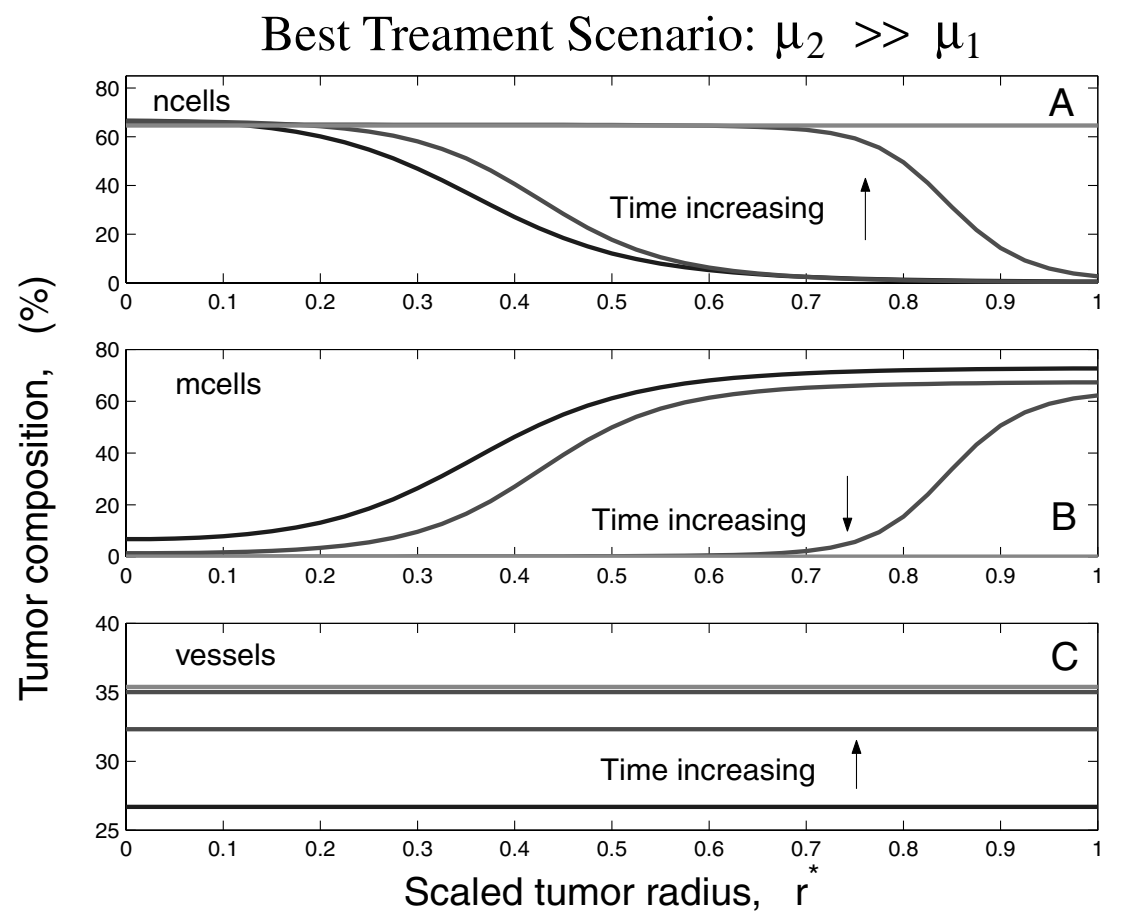

Fig. 8. Plot of temporal changes in tumor composition during the best treatment scenario, i.e. $\mu_{1}, \mu_{2} \gg 1$. The percentage of the tumor made up of drug-sensitive cells (A), semi-drug resistant cells (B), and blood vessels (C) are plotted against the scaled tumor radius.

on chemotherapeutic treatment is shown. The graph shows that when the best treatment scenario for each case is used, control of the tumor is significantly reduced when the vessel density changes dynamically.

\subsection{Effect of different vascular distributions on tumor response to therapy.}

If the tumor is entirely avascular, $v=\Gamma=0$, and the drug can only enter the tumor via diffusion from the surrounding normal tissue. In this case, it takes longer for the drug to accumulate in the tumor and, hence, the response is delayed. Equally, no drug is able to escape back into the vasculature, so that the positive effects of therapy may eventually outweigh those of constant vascular support.

In order to investigate the effect of a spatially dependent vascular support system we now take

$$
\begin{gathered}
v_{0}(r)=v_{0} H\left(r-R_{V}\right)= \begin{cases}v_{0} & R_{V}<r<R \\
0 & 0<r<R_{V}\end{cases} \\
\Gamma(r)=\Gamma H(v)= \begin{cases}\Gamma & 0<v \\
0 & v=0\end{cases}
\end{gathered}
$$

as the initial vascular density and blood-tissue transfer coefficient. This localizes the vasculature to the outer edge of the tumor, in an annulus of width $R-R_{V}$, 


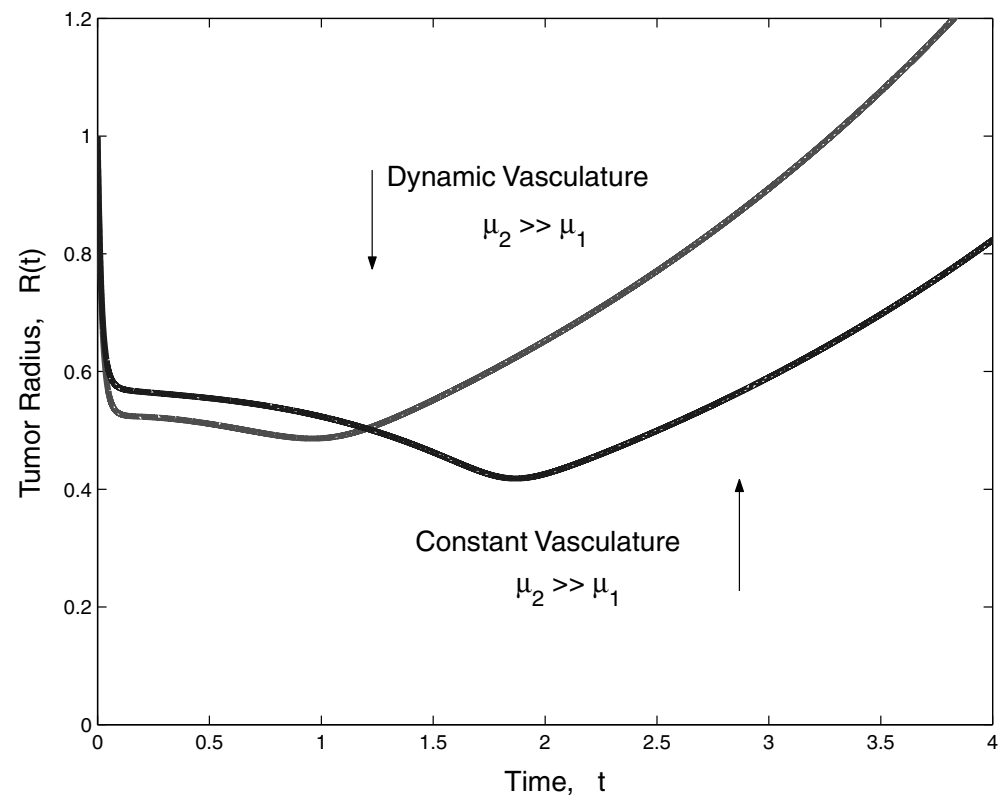

Fig. 9. Comparison of tumor response to chemotherapy when the volume fraction of vessels remains constant and is allowed to vary. The upper curve represents a tumor with dynamic vasculature given by (66) when the competition parameters are those for the best tumor response, i.e. $\mu_{2} \gg \mu_{1}$ (see Figure 7). The initial conditions for this case are $n_{0}=$ $0.75, m_{0}=0.15, v_{0}=0.1$. The lower curve depicts a tumor with a constant vascular proportion $\left(v<v^{*}=0.34\right)$ when the competition parameters are those for the best tumor response, i.e. $\mu_{2} \gg \mu_{1}$ (see Figure 4 ). The initial conditions for this case are $n_{0}=$ $0.75, m_{0}=0.15, v_{0}=0.1$.

leaving the inner core initially avascular. Note that with this choice of $v_{0}(r)$ there is no vascular exchange when the tumor is sufficiently small (i.e. $0<R<R_{V}$ ). The results presented in Figure 10 demonstrate the effect of incorporating these functions with $R_{v}=0.8 \mathrm{~cm}$ into equations (1) and (66). When the same parameter values from the previous section are used, simulations show that when the vascular density depends on radial position, the tumor's reduction in response to treatment is superior to that of an avascular tumor, however, the post-treatment re-growth rate is also much faster.

Figure 11 demonstrates another interesting result of a spatially varying vascular supply. When the spatially dependent initial conditions is used, the final tumor composition changes dramatically. In this case instead of the drug-sensitive cells eventually taking over the tumor, we have the drug-resistant population becoming dominant.

\section{Discussion}

In this paper, previous mathematical models that describe the chemotherapeutic response of vascular tumors are extended, to include polyclonality, direct cellular competition, and dynamic vascular support. The model is formulated as a system 


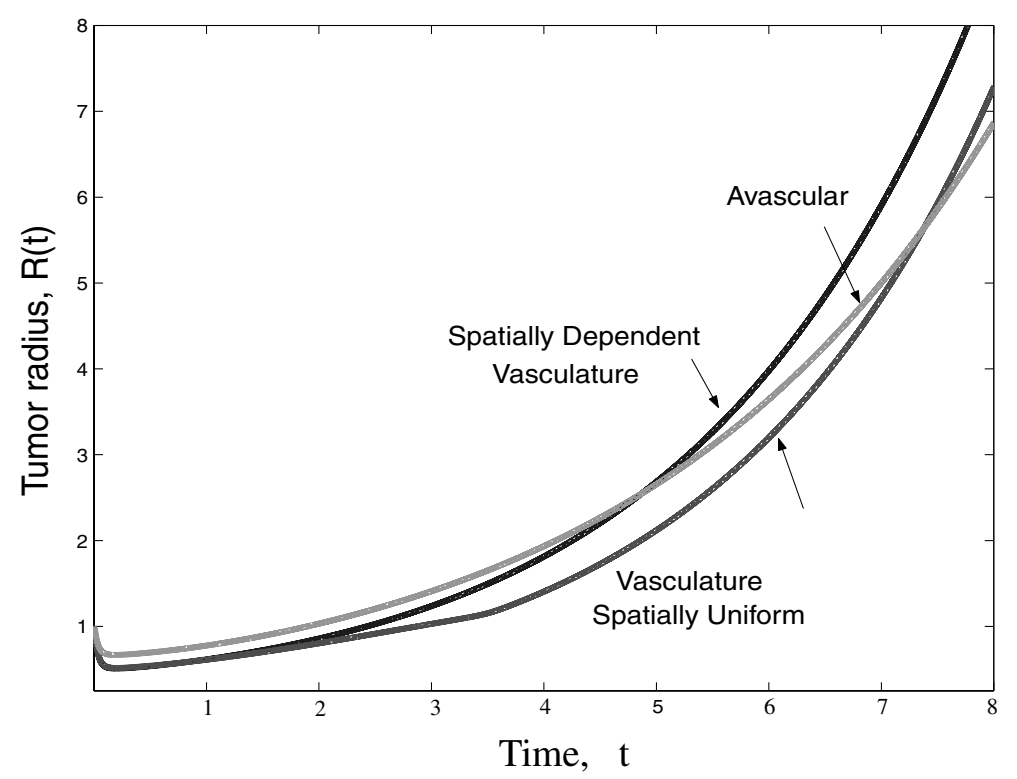

Fig. 10. Comparison of tumor response to chemotherapy when the volume fraction of vessels is zero, representing an avascular tumor; initially spatially-dependent; and spatially uniform throughout the tumor. Simulations are for the case when both drug-sensitive and resistant cells have a strong competitive effect on each other.

of partial differential equations that describe the evolution of the drug and cell populations within the tumor. The tumor radius is tracked by introducing a velocity field to describe cell motion generated by the balance between cell proliferation and death and viable blood vessel production and destruction. A combination of numerical and analytical techniques were used to investigate the tumor's response to traditional chemotherapeutic strategies and to contrast the behavior of tumors containing semi-drug resistant populations.

When the tumor consisted of only drug-sensitive cells and a constant vascular support system, it was possible to derive radially symmetric solutions and to determine the stability of these solution to asymmetric perturbations. The growth rates of the asymmetric modes were positive in the vicinity of the steady state corresponding to control of the tumor for a finite range of modes. This result suggests intratumoral drug concentrations which are adequate to control the growth of spherical tumors may be inadequate when asymmetries are present. The unstable modes represent the unsuccessful treatment and invasive growth of an asymmetric tumor under the same conditions which resulted in the control of a spherical tumor.

The emergence of drug resistance in a previously drug-sensitive tumor is thought to be one of the major barriers to effective chemotherapeutic treatment [5]. Biochemical or phenotypic resistance may arise in cells via spontaneous mutation [23] or be acquired as a result of exposure to the drug [15]. Notable differences in the tumor's response to therapy were observed when a second, drug resistant cell pop- 


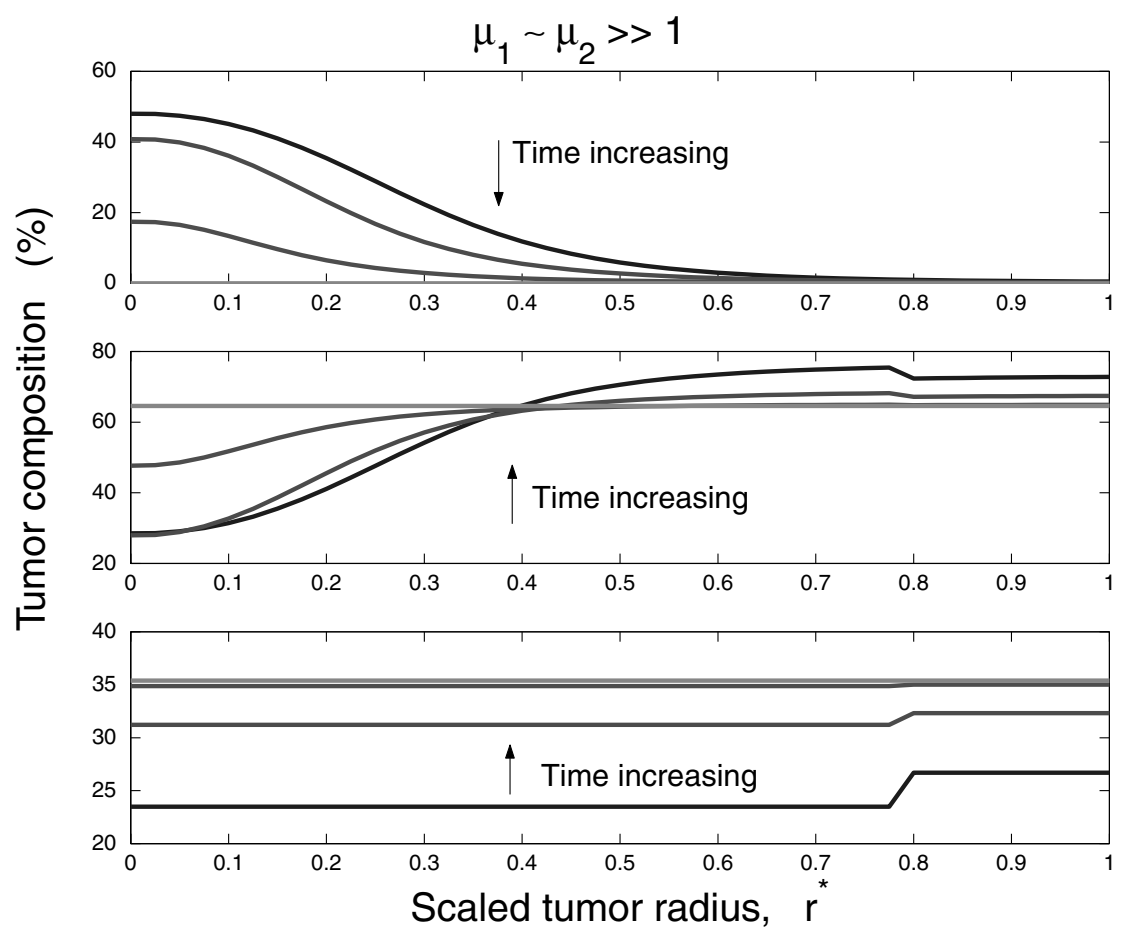

Fig. 11. Plot of the tumor composition when $\mu_{1}, \mu_{2} \gg 1$ and the vascular supply is initially spatially dependent. The percentage of the tumor made up of drug-sensitive cells (A), semidrug resistant cells (B), and blood vessels (C) is plotted against the scaled tumor radius for various times (1-4weeks).

ulation in direct competition with the drug sensitive cells was incorporated into the model. When the competitive effect of the sensitive cells on the resistant cells is much stronger than the resistant cells' competitive effect, the tumor's response to therapy is significantly improved over the case when there is no direct competition or equally strong competitive effects.

When the vascular density is allowed to increase/decrease according to cell density, we found that the best treatment scenario for prolonged tumor reduction corresponds to the drug-sensitive cells having a much stronger competitive effect on the drug-resistant cell population. However, the slowest tumor re-growth post treatment occurs when both cell types have equally large competition parameters. The results also highlight the dramatic increase in the tumor's re-growth rate when a dynamic vascular support system is included.

The modeling framework developed in [28] and extended in the current study leads naturally to several possibilities for further investigation. For example a mathematical investigation into the effectiveness of more sophisticated treatment strategies (such as direct or two-step drug targeting) in homogeneous or multi-cell type tumors could provide insight into the conditions for which one strategy is better 
than another. Further, there is no mention in the present model of necrotic cell death, hypoxia, rate of progression through the cell cycle or cell cycle arrest. Including these phenomena could have significant effects on the results presented here.

Small, avascular spheroids depend upon the formation of new blood vessels from the surrounding vasculature (angiogenesis) to make the transition from small and harmless to large and malignant [20], [26]. Anti-angiogenic drugs stop new vessels from forming and break up the existing vascular network that feeds the tumor. While in the present paper we have focused on traditional chemotherapeutic treatment strategies, the effects of anti-angiogenic drugs on the tumor vasculature and the dynamics of vascular shunting, collapse, and re-growth are interesting directions for future work which could be addressed by modification of this modeling framework. For example, [27] demonstrated that solid stress inhibits tumor growth in vitro, regardless of host species, tissue of origin, or differentiation state. The inhibiting stress for multicellular tumor spheroid growth in agarose matrices was 45 to $120 \mathrm{~mm} \mathrm{Hg}$, which greatly exceeds blood pressure is sufficient to the collapse blood vessels of in vivo tumors. The study of how this would effect drug delivery is an open problem.

The model presented has been able to predict the chemotherapeutic response of vascular tumors with a dynamic vascular support system and two distinct clonal populations in direct competition. The formulation of the model is general enough that more of the tumor physiology can be included and more sophisticated treatment strategies can be investigated so that improved protocols for chemotherapeutic treatment can be determined.

\section{References}

1. Adam, J.A.: A mathematical model of tumor growth. II Effects of geometry and spatial uniformity on stability, Math. Biosci. 86, 183 (1987)

2. Adam, J.A.: A mathematical model of tumor growth III: Comparison with experiment. Math. Biosci. 86, 213 (1987)

3. Adam, J.A., Maggelakis, S.A.: Diffusion regulated growth characteristics of a spherical prevascular carcinoma, Bull. Math. Biol. 52, 549 (1990)

4. Baxter, L.T., Yuan, F., Jain, R.K.: Pharmacokinetic analysis of the perivascular distribution of bifunctional antibodies and haptens: comparison with experimental data, Cancer Res. 52, 5838 (1992)

5. Birkhead, B.G., Rankin, E.M., Gallivan, S., Dones, L., Rubens, R.D.: A mathematical model of the development of drug resistance to cancer chemotherapy, Eur. J. Cancer Clin. Oncol. 23, 1421 (1987)

6. Byrne, H.M., Chaplain, M.A.J.: Growth of non-necrotic tumors in the presence and absence of inhibitors, Math. Biosci. 130, 151-181 (1995)

7. Byrne, H.M., Chaplain, M.A.J.: Growth of necrotic tumors in the presence and absence of inhibitors, Math. Biosci. 135, 187 (1996)

8. Byrne, H.M., Gourley, S.A.: The role of growth factors in avascular tumour growth, Math. Comput. Modelling 26, 83 (1997)

9. Byrne, H.M., Chaplain, M.A.J.: Free boundary value problems associated with the growth and development of multicellular spheroids, Eur. J. Appl. Math. 8, (1997) 639

10. Byrne, H.M., Chaplain, M.A.J.: Necrosis and apoptosis: distinct cell loss mechanisms in a mathematical model of avascular tumor growth, J. Theor. Med. 1, 223 (1998) 
11. Byrne, H.M.: A weakly nonlinear analysis of a model of avascular solid tumour growth, J. Math. Bio. 39, 59 (1999)

12. Byrne, H.M., Chaplain, M.A.J.: Modelling the role of cell-cell adhesion in the growth and development of carcinoma Math. Comput. Model. 24(12), 1 (1996)

13. Chaplain, M.A.J., Orme, M.E.: Travelling waves arising in mathematical models of tumour angiogenesis and invasion FORMA 10, 147 (1996)

14. Crank, J.: Free and moving boundary problems, O.U.P., 1988

15. Crowther, D.: A rational approach to the chemotherapy of human malignant disease--II, Brit. Med. J. 4, 216 (1974)

16. Curry, F.E., Mason, J.C., Michel, C.C.: Osmotic reflection of coefficients of capillary to low molecular weight hydrophilic solutes measures in single perfused capillaries of the frog mesentery, J. Physiol. London 261, 319 (1976)

17. Deen, W.M.: Hindered transport of large molecules in liquid-filled pores, AIChE J. 33, 1409 (1987)

18. Dordal, M.S., Winter, J.N., Atkinson, A.J.: Kinetic Analysis of P-glycoprotein-mediated Doxorubicin Efflux, J. Pharmacol. Exp. Ther. 263, 762 (1992)

19. Hahnfeldt, P., Panigrahy, D., Folkman, J., Hlatky, L.: Tumor development under angiogenic signaling: A dynamic theory of tumor growth, treatment response, and postvascular dormancy, Cancer Res. 59, 4770 (1999)

20. Folkman, J.: Fighting cancer by attacking its blood supply, Sci. Amer. Sept. 150 (1996)

21. Folkman, J., Hochberg, M.: Self-regulation of growth in three dimensions, J. Exp. Med. 138, 743 (1973)

22. Gerlowski, L.E., Jain, R.K.: Microvascular permeability of normal and neoplastic tissues, Micro. Vasc. Res. 31, 288 (1986)

23. Goldie, J.H., Coldman, A.J.: A mathematical model for relating the drug sensitivity of tumors to their spontaneous mutation rate, Cancer Treat. Rep. 63, 1727 (1971)

24. Greenspan, H.P.: Models for the growth of a solid tumor by diffusion, Stud. Appl. Math. 52, 317 (1972)

25. Greenspan, H.P.: On the growth and stability of cell cultures and solid tumors, J. Theor. Biol. 56, 229 (1976)

26. Hanahan, D., Folkman, J.: Patterns and emerging mechanisms of the angiogenic switch during tumorigenesis, Cell 86, 353 (1996)

27. Helmlinger, G., Netti, P.A., Lichtenbeld, H.C., Melder, R.J., Jain, R.K.: Solid stress inhibits the growth of multicellular tumour spheroids, Nature Biotech 5, 778 (1997)

28. Jackson, T.L., Byrne, H.M.: A mathematical model to study the effects of drug resistance and vasculature on the response of solid tumors to chemotherapy, Math. Biosci. 164, 17 (2000)

29. Jain, R.K.: Barriers to drug delivery in solid tumors, Sci. Amer. 271, 58 (1994)

30. Baxter, L.T., Jain, R.K.: Transport of fluid and macromolecules in tumors I. Role of interstitial pressure and convection, Microvasc. Res. 37, 77 (1989)

31. Jain, R.K.: Transport of Macromolecules Across Tumor Vasculature, Cancer Metastasis Rev. 6, 559 (1987)

32. Kerr, D.E., Schreiber, G.J., Vrudhula, V.M., Svensson, H.P., Hellstrom, K.E., Senter, P.D.: Regressions and cures of melanoma xenografts following treatment with monoclonal antibody $\beta$-lactamase conjugates in combination with anti-cancer prodrugs, Cancer Res. 55, 3558 (1995)

33. Kerr, J.F.R., Wyllie, A.H., Currie, A.R.: Apoptosis: a basic biological phenomenon with wide-ranging implications in tissue kinetics, Br. J. Cancer 26, 239 (1972)

34. Kunz-Schughart, L.A., Kreutz, M., Kneuchel, R.: Multicellular spheroids: a threedimensional in vitro culture system to study tumor biology, Int. J. Eng. Path. 79, 1-23 (1998) 
35. Liotta, L.A., Saidel, G.M., Kleinerman, J.: Diffusion model of tumor vascularization, Bull. Math. Biol. 39, 117 (1977)

36. Maggelakis, S.A., Adam, J.A.: Mathematical model of prevascular growth of a spherical carcinoma, Math. Comput. Modelling 13, 23-38 (1990)

37. Marusic, M., Bajzer, Z., Vuk-Pavlovic, S., Freyer, J.P., Tumor growth in vivo and as multicellular spheroids compared by mathematical models, Bull. Math. Biol. 56, 617 (1994)

38. Nugent, L.J., Jain, R.K.: Extravascular diffusion in normal and neoplastic tissues, Cancer Res. 44, 238 (1984)

39. O’Reilly, M.S., Boehm, T., Shing, Y., Fukai, N., Vasios, G., Lane, W.S., Flynn, E., Birkhead, J.R., Folkman, J.: Endostatin: An endogenous inhibitor of angiogenesis and tumor growth, Cell 88, 277 (1997)

40. Orme, M.E., Chaplain, M.A.J.: A mathematical model of vascular tumor growth and invasion, Math. Comp. Model. 23(10), 43 (1996)

41. Panetta, J.C., Adam, J.: A mathematical model of cycle-specific chemotherapy, Math. Comput. Modelling 22, 67 (1995)

42. Panetta, J.C.: A mathematical model of periodically pulsed chemotherapy: Tumor recurrence and metastasis in a competitive environment, Bull. Math. Biol. 88, 425 (1996)

43. Robert, J., Illiadia, A., Hoerni, B., Cano, J., Durand, M., Lagarde, C.: Pharmacokinetics of Adriamycin in Patients with Breast Cancer: Correlation Between Pharmacokinetic Parameters and Clinical Short-term Response, Eur. J. Cancer Clin. Oncol. 18, 739 (1982)

44. Sutherland, R.M., Durand, R.E.: Growth and cellular characteristics of multicell spheroids, Recent Results in Cancer Research 95, 24 (1984)

45. Siemers, N.O., Kerr, D.E., Yarnold, S., Stebbins, M., Vrudgyka, V.M., Hellstrom, I., Hellstrom, K.E., Senter, P.D.: L49-sFv- $\beta$-Lactamase, a single chain anti-p97 antibody fusion protein, Bioconjugate Chem. 8, 510 (1997)

46. Ward, J.P., King, J.R.: Mathematical modeling of avascular-tumor growth, IMA J. Math. Appl. Med. Biol. 14, 39 (1997) 